\title{
An Adaptive Multi-User MIMO Scheme for the Millimeter-Wave Downlink
}

\author{
Siyao Lu, Ibrahim A. Hemadeh, Mohammed El-Hajjar, Lajos Hanzo \\ School of Electronics and Computer Science, University of Southampton, UK. \\ Email: $\{$ sl18e14, meh, lh $\} @$ ecs.soton.ac.uk
}

\begin{abstract}
Space-time shift keying (STSK) constitutes a beneficial multiple-input-multiple-output (MIMO) technique that strikes a compelling trade-off between the multiplexing and diversity gains attained. Its close relative, namely layered multi-group STSK (LMG-STSK) combines the concepts of multi-user MIMO, STSK and beamforming for simultaneously conveying information to multiple users. Generalized frequency division multiplexing (GFDM) has been proposed as a promising multicarrier modulation candidate for dispersive wideband channels since it benefits from its time-frequency domain structure when combined with a powerful MIMO scheme. As a further evolution, we propose a novel GFDM-aided adaptive layered multi-group STSK (LMGSTSK) system for the downlink of millimeter wave communications systems. The proposed system tackles the propagation challenges of the high pathloss at millimeter wave frequencies, where multiple users are clustered into groups served by different antenna layers at the transmitter. We propose an adaptive scheme that can adapt both the number of transmit antennas (TAs) per layer based on the number of users in each user group in order to maximize the number of users served and also the STSK codeword in order to maximise the achievable user throughput.
\end{abstract}

\section{INTRODUCTION}

Millimeter-wave (mmWave) communications have been developed for tackling the global bandwidth shortage by harvesting the wide license-free bandwidth [1] [2]. However, mmWave systems suffer from both high attenuation and sparse multipath scattering, when communicating over a frequency-selective channel [3] [4]. Therefore, it is crucial for mmWave systems to be combined with MIMO and multi-carrier techniques for overcoming the unfavorable propagation charateristics, as well as for improving the reliability and increasing the data rates attained. Finally, the high pathloss of mmWave frequencies makes beamforming (BF) a key enabling technology [5] [6].

Given the short wavelength, say $0.5 \mathrm{~cm}$ at $60 \mathrm{GHz}$, a large number of antennas can be accommodated at the transmitter and receiver in a compact space and different MIMO gains such as diversity and/or multiplexing gains as well as beamforming gains, can be achieved by MIMO techniques due to the increased degrees of freedom in the system [4] [5]. Combining different MIMO gains in the context of multifunctional MIMOs, has been presented in [7] [8]. As a further development, spatial modulation (SM) was proposed in [9], which is capable of striking a compelling performance versus complexity trade-off with the aid of a single radio frequency (RF) chain. Historically speaking, the idea of SM was also combined with linear dispersion coding (LDC) [9] and STSK [10] [11] for striking a flexible diversity-multiplexing tradeoff, which is capable of overcoming the high pathloss with the aid of beamforming.

The concept of LMG-STSK has been proposed in [12] for mmWave communications, which clusters the users supported into groups and also partitions the TAs into several layers, where each layer serves one of the user groups. Each TA layer serves a specific group of users, where the users in each group all fall within the same angularly selective transmitted beam, but they are still sufficiently seperated by several wavelengths within the beam for experiencing independent fading channels. As a benefit, the application of LMG-STSK tackles the challenge of multi-user MIMO techniques at mmWave frequencies, where the base station (BS) cannot readily communicate with angularly seperated users at the same time due to having a narrow transmit beam.

Adaptive modulation and coding techniques have been conceived for significantly increasing the achievable data rate, reliability and spectrum efficiency of wireless communications by promptly adapting the system parameters in order to achieve a specific quality of service (QoS) [8] [13]. When employing adaptation, the most appropriate coding and modulation scheme is activated under rapidly-fluctuating channel conditions, which results in a higher effective throughput than its non-adaptive counterpart, while maintaining the required QoS, such as say the target bit-error-ratio (BER).

In this paper, we conceive a novel adaptive LMG-STSK system operating at mmWave frequencies combined with GFDM [14] [15], which has been proposed as a flexible multi-carrier transmission technique that provides additional degrees of freedom compared to classic orthogonal frequency division multiplexing (OFDM). The novel contributions of this paper are as follows:

- We propose a novel GFDM-aided adaptive LMG-STSK system for the mmWave downlink in order to increase both the number of users supported and the individual user throughput, while maintaining a defined QoS.

- Two levels of adaptation are employed. Firstly, the proposed system adaptively configures the TAs for each antenna layer depending on the number of users per group, which is time-variant in a mobile environment. The criterion used for adapting the number of antennas per layer is to keep the diversity order of all users in all groups similar. Secondly, in order to increase the user throughput in each group, with the diversity order fixed by the number of antennas per group, the STSK codewords of each group can also be adapted by switching among several STSK encoders according to the channel signal-tonoise ratio (SNR) conditions, while maintaining the target BER.

The paper is organised as follows. In Section II, we introduce the architecture of the proposed GFDM-aided adaptive LMG-STSK system. Then our simulation results will be provided in Section III and finally we conclude in Section IV. 


\section{Proposed GFDM-Aided AdAPTIVE LMG-STSK SYSTEM}

In this section we introduce the novel multi-user GFDMaided adaptive LMG-STSK system's architecture proposed for downlink transmission in mmWave communications, where the low-complexity GFDM modem design of [16] will be utilised in the proposed adaptive system.

Due to the significant propagation losses of the mmWave channel, high-gain directional beamforming should be used for compensating the high attenuation [5] [11]. However, not all users of a multi-user system will fall in the coverage angle of a transmitted beam. Therefore, partitioning the users into different user groups is an efficient way for the BS to communicate with more users at a time. The LMG-STSK technique proposed in [12] partitions the TAs into antenna layers and each layer serves a specific group of users, which allows more users to be served simultaneously in the downlink over the same time and frequency resources.

The system proposed in [12] divided the total number of TAs equally among each antenna layer for serving a specific user group without considering the fluctuating number of users per group due to mobility. Explicitly, in practice the number of users in a group varies, since the users tend to be mobile, where a user may traverse from one user group to another or a user may move to an out-of-range location. If the same number of antennas is assigned to all antenna layers, the groups supporting less users benefit from higher diversity order due to the additionally assigned TAs, while the groups having more users than the maximum possible number of users per group may not be able to serve all users at the same time. Therefore, we propose an adaptive LMG-STSK system that can adaptively assign TAs according to the number of users in the user group instead of simply considering the same number of TAs for all user groups. As defined in [10], the STSK system is specified by the parameters $(M, N, T, Q, L)$, where the throughput per STSK codeword can be expressed as

$$
R_{c}=\log _{2}(Q L),
$$

while $M$ and $N$ indicate the number of TAs and receive antennas (RAs), respectively, $T$ is the number of time slots, $Q$ is the number of dispersion matrices and $L$ represents $L$ ary modulation. Hence, the achievable user throughput can be changed by applying different number of dispersion matrices $Q$ or different constellations sizes $L$ in conjunction with the fixed values of $M, N$ and $T$.

Therefore, we consider two levels of adaptation in our proposed GFDM-aided LMG-STSK downlink system as follows:

- Firstly, the number of TAs assigned to different antenna layers is adapted according to the time-variant number of users in a user group, while arranging for the users in all groups to have similar diversity orders. The diversity order of the $n_{u}$-th user in the $n_{g}$-th group can be calculated as [12]:

$$
\mathcal{D}=\left(N_{g}^{t}-\sum_{i \neq n_{u}}^{N_{g}^{u}} N_{g}^{r, i}\right) \times \min (M, T),
$$

where $i$ is the index of users in the $n_{g}$-th group. For example, let us assume that the BS is equipped with 32 number of TAs to serve user groups. These antenna layers serve
4 specific user groups of size $(2,6,3,5)$, respectively. Based on the diversity order of (2), the BS adaptively assigns $(4,12,6,10)$ TAs to serve the corresponding user groups, while maintaining a similar diversity orders for all users in all groups, where the diversity orders in this example are $\mathcal{D}=(4-2 \times 1) \times \min (2,2)=4$. Then, assuming that the number of users in these groups changes to $(2,5,4,4)$, the BS assigns $(5,11,8,8)$ TAs to serve the specific user groups, where the diversity orders of the individual users in these 4 user groups are $(6,6,4,4)$, respectively.

- Secondly, given the number of antennas per antenna layer as set in the first step above, the adaptive system increases the individual user throughput by switching between different STSK codewords, which are uniquely specified by the different combinations of $Q$ and $L$, while always satisfying the target BER performance. The receiver of the adaptive system estimates the SNR of the received signal. Then, the SNR is fed back to the transmitter and then the transmitter makes a decision on the STSK codeword activated by searching through the look-up tables according to the SNR.

The procedures used at the transmitter will be detailed in Section II-A and the signal processing used at the receiver will be discussed in Section II-B.

\section{A. Transmitter}

We consider the downlink of our GFDM-aided adaptive LMG-STSK system shown in Fig. 1, where a transmitter is equipped with $N^{t}$ TAs and communicates with $N_{G}$ user groups, where the number of users in each group is timevariant. Before transmission, the transmitter partitions $N_{u}$ users into $N_{G}$ user groups and then $N_{u}$ STSK encoders and GFDM modulators are assigned to the corresponding user groups as shown in Fig. 1. Furthermore, $N^{t}$ TAs are also grouped into $N_{G}$ antenna layers for communicating with the users in the corresponding user groups. Furthermore, $N_{g}^{t}$ TAs are assigned to the $n_{g}$-th antenna layer for serving $N_{g}^{u}$ number of users in the $n_{g}$-th user group, where the number of users in each user group is variable. The number of corresponding RAs of the $n_{u}$-th user in the $n_{g}$-th group is $N_{g}^{r, n_{u}}$ and each user group is isolated from the other group by applying highly directional beamforming for each antenna layer. The application of beamforming eliminates the interferences amongst the user groups. In Fig. 1, each STSK encoder is characterized by STSK $(M, N, T, Q, L)$, where $M, N$ and $T$ are fixed values in the adaptive system, while the values of $Q$ and $L$ are carefully controlled for adapting the different STSK codewords depending on the SNR fed back from the receiver to the transmitter. As seen in Fig. 1, after the adaptation of TAs in each antenna layer for serving the corresponding user groups and after the decisions concerning the choice of STSK codewords, the downlink source information designed for each user is entered into the STSK encoder, followed by the GFDM modulator. Then in order to avoid the multi-user interference (MUI) within a specific user group, where all users in a user group employ the same frequency, multi-user transmit precoding (MU-TPC) is applied to the users in the same user group after GFDM-STSK processing. The MU-TPC of our system is based on the classic block diagonalization (BD) 


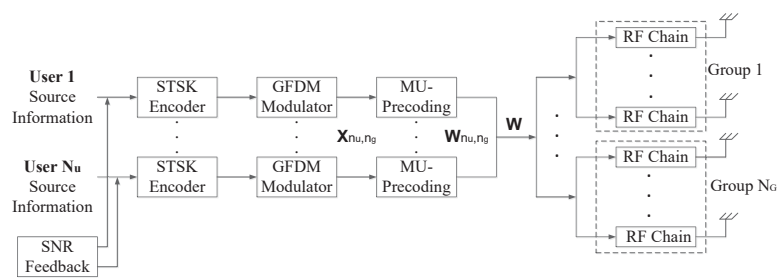

Fig. 1. The transmitter architecture of GFDM-aided adaptive LMGSTSK system. The TAs are adaptively assigned to $N_{G}$ user groups according to the specific number of users in the different groups.

technique $^{1}$ [11], which is a linear TPC technique designed for eliminating the MUI in a user group. The matrix $\mathbf{X}_{n_{u}, n_{g}}$ represents the data symbol matrix of the $n_{u}$-th user in the $n_{g}$-th group after GFDM-STSK processing, where we have $\mathbf{X}_{n_{u}, n_{g}} \in \mathbb{C}^{M \times N_{g f d m}}$, and $N_{g f d m}=\left(N_{s} \times T\right)$ that shows the number of GFDM-STSK data symbols over $T$ time slots in the $m$-th TA. For convenience, the GFDM modulated symbol vector $X_{m, n_{u}, n_{g}}[i]$ of the $n_{u}$-th user in the $n_{g}$-th group at the $m$-th TA can be expressed in the frequency domain as

$$
D_{m, n_{u}, n_{g}}[i]=\sqrt{\frac{1}{N_{g f d m}}} \sum_{n=0}^{N_{g f d m}-1} X_{m, n_{u}, n_{g}}[i] \mathrm{e}^{\frac{-j 2 \pi n i}{N_{g f d m}}},
$$

where $i=0, \ldots, N_{g f d m}-1$.

The MU-TPC matrix of the $n_{u}$-th user in the $n_{g}$-th group is represented as $\mathbf{P}_{M U}^{t, n_{u}, n_{g}}[i] \in \mathbb{C}^{N_{g}^{t} \times M}$. The data symbol matrix after MU-TPC is $\mathbf{W}_{n_{u}, n_{g}} \in \mathbb{C}^{N_{g}^{t} \times N_{g f d m}}$, which can be expanded as

$$
\mathbf{W}_{n_{u}, n_{g}}[i]=\mathbf{P}_{M U}^{t, n_{u}, n_{g}}[i] \mathbf{D}_{n_{u}, n_{g}}[i] .
$$

After MU-TPC for the users in the same group, the data symbols conveyed over $T$ time slots with the same frequencydomain index $i$ for all users in the $n_{g}$-th group can be written as

$$
\mathbf{W}_{n_{g}}[i]=\sum_{n_{u}=1}^{N_{g}^{u}} \mathbf{W}_{n_{u}, n_{g}}[i] .
$$

Hence, the corresponding data symbols at the $i$-th subcarrier index after MU-TPC for all users in all groups can be represented by

$$
\mathbf{W}[i]=\left[\mathbf{W}_{1}[i], \mathbf{W}_{2}[i], \ldots, \mathbf{W}_{n_{g}}[i], \ldots, \mathbf{W}_{N_{G}}[i]\right]^{T} .
$$

Then the data symbols $\mathbf{W}[i]$ are mapped to $N_{G}$ antenna layers for simultaneous transmission from $N^{t}$ TAs over $T$ time slots. Before transmission, each symbol matrix $\mathbf{W}_{n_{g}}$ is transformed to the time-domain representation of the data symbols. Therefore, the data symbols in the $n_{g}$-th group at the $n_{g}^{t}$-th TA are represented as

$$
\bar{W}_{n_{g}, n_{g}^{t}}[i]=\sqrt{N_{g f d m}} \sum_{n=0}^{N_{g f d m}-1} W_{m, n_{g}, n_{g}^{t}}[i] \mathrm{e}^{\frac{j 2 \pi n i}{N_{g f d m}}}
$$

\footnotetext{
${ }^{1}$ Any other MU-TPC techniques can be employed in the proposed system, but we use the BD for simplicity.
}

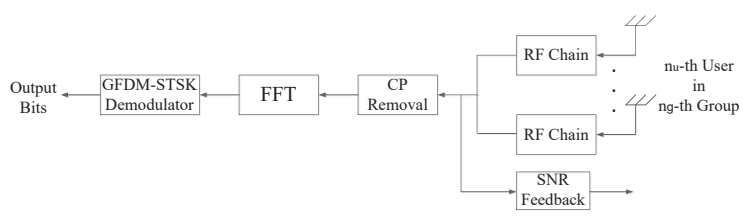

Fig. 2. The receiver block diagram of GFDM-aided adaptive LMGSTSK system for the $n_{u}$-th user in the $n_{g}$-th group received from $N_{A E}^{r}$ number of RA arrays.

where $i=0, \ldots, N_{g f d m}-1$.

The cyclic prefix (CP) of length $L_{c p}$ is concatenated to the symbols vector $\overline{\mathbf{W}}_{n_{g}, n_{g}^{t}}$ for protecting the data from dispersion. After CP insertion, the data symbol matrix of each antenna layer becomes $\widehat{\mathbf{W}}_{n_{g}} \in \mathbb{C}^{N_{g}^{t} \times\left(N_{g f d m}+L_{c p}\right)}$.

Then analogue beamforming $(\mathrm{ABF})$ invoked for seperating the different user groups and each antenna layer can be designed to its intended user group. The $\mathrm{ABF}$ matrix of the $n_{g}$-th user group is represented by $\mathbf{B}_{n_{g}}^{T x}[i] \in \mathbb{C}^{N_{A E}^{t, n_{g}} \times N_{g}^{t}}$, where $N_{A E}^{t, n_{g}}$ is the number of TA elements in $n_{g}$-th layer, $i=\left(0, \ldots,\left(N_{g f d m}+L_{c p}\right)-1\right)$. As a result, the final transmitted symbols after ABF destined for the $n_{g}$-th user group can be represented as

$$
\mathbf{S}_{n_{g}}[i]=\mathbf{B}_{n_{g}}^{T x}[i] \widehat{\mathbf{W}}_{n_{g}}[i],
$$

where $i=0, \ldots,\left(N_{g f d m}+L_{c p}\right)-1$, and $\mathbf{S}_{n_{g}} \in$ $\mathbb{C}^{N_{A E}^{t, n g} \times\left(N_{g f d m}+L_{c p}\right)}$. The matrix $\mathbf{S}_{n_{g}}$ will be used in the rest of this paper to represent the time-domain transmitted signal of the $n_{g}$-th user group in our adaptive system and its frequencydomain counterpart is denoted as $\overline{\mathbf{S}}_{n_{g}}$.

\section{B. Receiver}

After transmission in the mmWave channel, the signal of the $n_{u}$-th user in the $n_{g}$-th user group is received by $N_{A E}^{r}$ number of RA arrays at the receiver, as shown in Fig. 2.

After CP removal, the received signal of the $n_{u}$-th user in the $n_{g}$-th user group in the frequency domain can be represented as:

$$
\begin{aligned}
\mathbf{R}_{n_{g}, n_{u}} & =\mathbf{H}_{n_{g}, n_{u}} \overline{\mathbf{S}}_{n_{g}, n_{u}}+\mathbf{N} \\
& =\mathbf{H}_{n_{g}, n_{u}} \mathbf{B}_{n_{u}, n_{g}}^{T x} \widehat{\mathbf{W}}_{n_{g}, n_{u}}+\mathbf{N},
\end{aligned}
$$

where $\mathbf{N}$ is additive white Gaussian noise (AWGN) matrix of size $\left(N_{A E}^{r} \times N_{g f d m}\right)$ and $\mathbf{H}_{n_{g}, n_{u}}$ is the mmWave channel matrix of the $n_{u}$-th user in the $n_{g}$-th user group. The channel model applied in this paper follows that of [3] and [17].

In the receiver block of Fig. 2, ABF is applied by using the beamforming weights $\mathbf{B}_{n_{u}, n_{g}}^{R x}$ for eliminating the interferences between different user groups and the received signal of the $n_{u}$-th user in the $n_{g}$-th group after $\mathrm{ABF}$ is represented by:

$$
\widetilde{\mathbf{R}}_{n_{g}, n_{u}}=\mathbf{B}_{n_{u}, n_{g}}^{R x} \mathbf{H}_{n_{g}, n_{u}} \mathbf{B}_{n_{u}, n_{g}}^{T x} \widehat{\mathbf{W}}_{n_{g}}+\tilde{\mathbf{N}} .
$$

The received signal $\widetilde{\mathbf{R}}_{n_{g}, n_{u}}$ of the $n_{u}$-th user in the $n_{g}$-th group shown in (10) has no interference contributions from the other users in the $n_{g}$-th group, which in a benefit of the MU-TPC employed at the transmitter. Furthermore, there is no interference between the different user groups due to the use of $\mathrm{ABF}$. 
TABLE I

SYSTEM PARAMETERS

\begin{tabular}{|l|l|}
\hline Parameters & Values \\
\hline Multi-carrier Scheme & GFDM (Low Complexity) \\
\hline Number of Subcarriers $N_{c}$ and $L_{c p}$ & 2048 and 90 \\
\hline STSK Encoder $(M, N, T, Q, L)$ & $(2,2,2,2,4)$ \\
& $(2,2,2,16,2)$ \\
& $(2,2,2,4,16)$ \\
\hline GFDM-STSK Frames & 1000 \\
\hline Total TAs, $N^{t}$ & 32 \\
\hline Total User Groups, $N_{G}$ & 4 \\
\hline Bandwidth and Carrier Frequency & $500 \mathrm{MHz}$ and $28 \mathrm{GHz}$ \\
\hline
\end{tabular}

Afterwards, according to (10), we represent the channel of the $n_{u}$-th user in the $n_{g}$-th group as an effective channel as follows:

$$
\widetilde{\mathbf{H}}_{n_{g}, n_{u}}=\mathbf{B}_{n_{u}, n_{g}}^{R x} \mathbf{H}_{n_{g}, n_{u}} \mathbf{B}_{n_{u}, n_{g}}^{T x} .
$$

Then frequency domain equalization (FDE) is used for compensating the impairments caused by the high pathloss encountered at mmWave frequencies. In this paper, the zero forcing (ZF) FDE [11] [16] is utilized at each subcarrier frequency. The equalized signal $\overline{\mathbf{R}}_{n_{g}, n_{u}}$ based on the effective channel matrix is obtained, which is then fed into the GFDM demodulators over $T$ time slots. At the GFDM demodulators of Fig. 2, the low-complexity design introduced in [16] is employed. After GFDM demodulation, the demodulated symbols obeying our carefully designed time-frequency structure are obtained. Then, the space-time symbol at the $n_{c}$-th subcarrier is decoded and detected by the STSK decoder for recovering the information bits of the $n_{u}$-th user in the $n_{g}$-th group.

\section{Simulation Results}

In this section we provide our performance results for characterizing the proposed GFDM-aided adaptive LMG-STSK system in mmWave channels. The performance is evaluated by using Monte Carlo simulations. We assume perfect synchronization and perfect channel knowledge both at the transmitter and receiver. The simulation parameters of the proposed adaptive system are listed in Table I. Furthermore, the system is simulated under the assumption that no $\mathrm{ABF}$ is applied for the sake of simplifying our analysis, although the employment of $\mathrm{ABF}$ is compulsory. The BER performance will be further enhanced with the aid of ABF. The adaptive system was designed to meet the target BER of $10^{-3}$.

In our simulations, we consider a BS equipped with $N_{t}=$ 32 TAs for operating at $28 \mathrm{GHz}$ carrier frequency assigned to 4 TA layers ${ }^{2}\left(G_{1}, G_{2}, G_{3}, G_{4}\right)$, where each antenna layer serves a specific user group. The number of users in each group is time-variant due to the movement of users, which is limited to $N_{G}^{\max }=\frac{N_{t}}{M}=16$ for all groups due to the use of the linear BD MU-TPC technique. Hence, the sum of users in the four user groups should be no more than 16 in the simulations. Upon considering a single user in a user group, both the assignment of TAs and the number of users in it directly influence the diversity order calculated in (2). Based on (2), when we assume that 32 TAs are assigned to an antenna layer for serving the user group of just 1 user, the diversity order for this single user is $\mathcal{D}=(32-0) \times \min (2,2)=64$,

${ }^{2}$ The proposed adaptive system is suitable for any number of TA layers and any number of user groups.

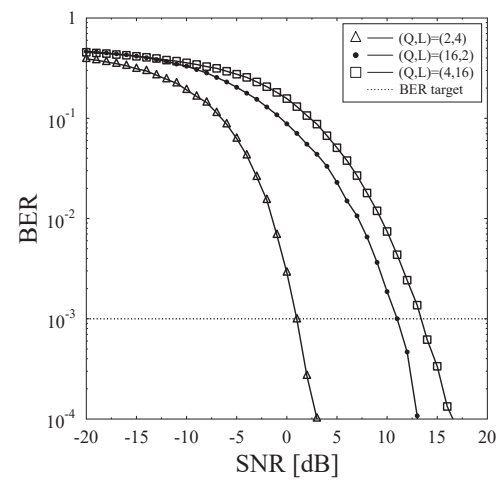

Fig. 3. BER performance of different STSK codewords for a fixed diversity order of $\mathcal{D}=6$. All other parameters are listed in Table I.

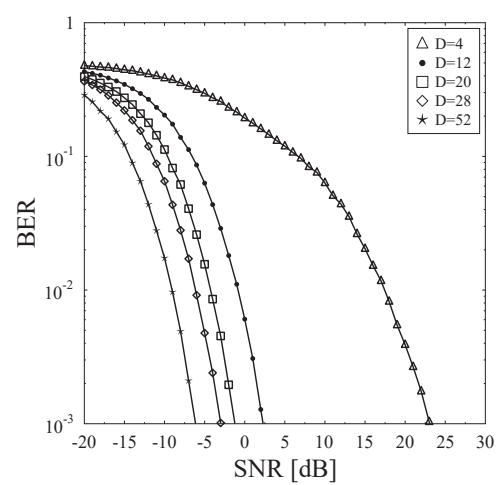

Fig. 4. BER performance of different diversity orders for the STSK $(2,2,2,16,2)$ encoder. All other parameters are listed in Table I.

which is the highest achievable diversity order of a user in a group. On the other hand, if we assume that 32 TAs are assigned to an antenna layer to serve the user group of the maximum of 16 users, then the diversity order of a user is $\mathcal{D}=(32-2 \times 15) \times \min (2,2)=4$, which is the lowest diversity order of a user in a group. Hence, the diversity order of a user in a group based on our simulation parameters varies from 4 to 64 . The BER performances of a single user relying on different $\operatorname{STSK}(2,2,2, Q, L)$ encoders is illustrated in Fig. 3 when considering a diversity order of $\mathcal{D}=6$. In the system characterized in Fig. 3 we consider 4 users in group $G_{1}$ and 9 TAs are assigned to this group, where a diversity order of $\mathcal{D}=6$ is obtained for a user in this group. The three STSK encoders of, $(2,2,2,2,4),(2,2,2,16,2)$ and $(2,2,2,4,16)$ associated with 3 bits/symbol, 5 bits/symbol, and 6 bits/symbol user throughputs, respectively, are considered. As shown in Fig. 3, upon fixing the same diversity order of $\mathcal{D}=6$, in order to maintain the target BER of $10^{-3}$, the system having higher user throughputs requires higher SNR values to reach the BER target.

In Fig. 4, the STSK $(2,2,2,2,4)$ encoder is employed and five different diversity orders are investigated, where the BER 


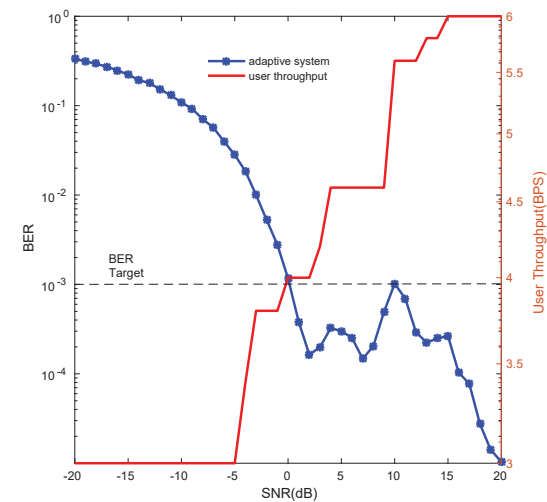

Fig. 5. BER performance of the GFDM-aided adaptive LMG-STSK system and the user throughput for a target BER of $10^{-3}$. All other parameters are listed in Table I.

performance is enhanced upon increasing the diversity order. Hence, in order to maintain the target BER, our LMG-STSK systems having different diversity orders require different SNR values. Therefore, in order to avoid simulating the LMG-STSK systems with all possible diversity order values, we devised a closed-form function to relate the SNRs of the different configurations attaining different diversity orders, in order to maintain the target BER. According to the SNRs at the target BER, we can get a simple numerical expression between the SNRs and diversity orders applying the $\operatorname{STSK}(2,2,2,16,2)$ scheme. Each STSK codeword in our simulations has a corresponding closed-form function. As a result, we can find the SNR values at the BER target for each diversity order for the same STSK codeword. In the proposed adaptive system, the receiver estimates the SNR of the received signal and then the SNR is fed back to the transmitter. The transmitter decides the choice of the most appropriate STSK codeword by searching through the look-up table for each diversity order using the formulas inferred.

Fig. 5 shows the BER performance of the proposed adaptive system, as well as the user throughput improvement attained. A uniformly-distributed random number of users is generated and the users are assigned into four groups. The 32 TAs are then assigned to these four groups. Again, the per-group antenna assignment criterion is to ensure that each user's diversity order is similar in all groups, where the calculation of diversity order follows (2). Having determined the diversity order, the STSK scheme is then adapted depending on the channel quality, which is quantified in terms of the instantaneous channel SNR. Given the channel SNR knowledge, the transmitter can choose the most suitable STSK codeword for maintaining the target performance. Therefore, the adaptive system characterized in Fig. 5 maintains the target BER, while gradually increasing the achievable user throughput within the group.

\section{CONCLUSIONS}

In this paper, a GFDM-aided adaptive multi-user MIMO system was proposed for downlink transmissions. The system increases the MU-MIMO efficiency at mmWave frequencies by carefully grouping all users and appropriately aportioning the TAs for each user group, which simultaneously adapting the number of TAs per layer based on the number of users in each user group, while increasing the achievable user throughput by switching among different STSK codewords.

\section{REFERENCES}

[1] T. S. Rappaport, S. Sun, R. Mayzus, H. Zhao, Y. Azar, K. Wang, G. N. Wong, J. K. Schulz, M. Samimi, and F. Gutierrez, "Millimeter wave mobile communications for $5 \mathrm{G}$ cellular: it will work!" IEEE Access, vol. 1, pp. 335-349, May 2013.

[2] I. Hemadeh, K. Satyanarayana, M. El-Hajjar, and L. Hanzo, "Millimeter-wave communications: physical channel models, design considerations, antenna constructions and link-budget," IEEE Communications Surveys Tutorials, vol. PP, no. 99, pp $1-1,2018$.

[3] M. R. Akdeniz, Y. Liu, M. K. Samimi, S. Sun, S. Rangan, T. S. Rappaport, and E. Erkip, "Millimeter wave channel modeling and cellular capacity evaluation," IEEE Journal on Selected Areas in Communications, vol. 32, no. 6, pp. 1164-1179, June 2014.

[4] Z. Pi and F. Khan, "An introduction to millimeter-wave mobile broadband systems," IEEE Communications Magazine, vol. 49, no. 6, pp. 101-107, June 2011.

[5] S. Sun, T. S. Rappaport, R. W. Heath, A. Nix, and S. Rangan, "MIMO for millimeter-wave wireless communications: beamforming, spatial multiplexing, or both?" IEEE Communications Magazine, vol. 52, no. 12, pp. 110-121, December 2014.

[6] K. Satyanarayana, M. El-Hajjar, P. H. Kuo, A. Mourad, and L. Hanzo, "Dual-function hybrid beamforming and transmit diversity aided millimeter wave architecture," IEEE Transactions on Vehicular Technology, vol. PP, no. 99, pp. 1-1, 2017.

[7] L. Hanzo, O. Alamri, M. El-Hajjar, and N. Wu, Near-Capacity Multi-Functional MIMO Systems. Wiley-Blackwell, 2009.

[8] L. Hanzo, M. El-Hajjar, and O. Alamri, "Near-capacity wireless transceivers and cooperative communications in the MIMO era: evolution of standards, waveform Design, and future perspectives," Proceedings of the IEEE, vol. 99, no. 8, pp. 1343-1385, Aug 2011.

[9] R. Y. Mesleh, H. Haas, S. Sinanovic, C. W. Ahn, and S. Yun, "Spatial modulation," IEEE Transactions on Vehicular Technology, vol. 57, no. 4, pp. 2228-2241, July 2008.

[10] S. Sugiura, S. Chen, and L. Hanzo, "A universal space-time architecture for multiple-antenna aided systems," IEEE Coтmunications Surveys Tutorials, vol. 14, no. 2, pp. 401-420, January 2012.

[11] Q. H. Spencer, A. L. Swindlehurst, and M. Haardt, "Zero-forcing methods for downlink spatial multiplexing in multiuser MIMO channels," IEEE Transactions on Signal Processing, vol. 52, no. 2, pp. 461-471, Feb 2004.

[12] I. A. Hemadeh, M. El-Hajjar, S. Won, and L. Hanzo, "Layered multi-group steered space-time shift-keying for millimeter-wave communications," IEEE Access, vol. 4, pp. 3708-3718, April 2016.

[13] L. Hanzo, S. X. Ng, T. Keller, and W. Webb, Quadrature amplitude nodulation. Wiley-IEEE Press, 2004, pp. 1036[Online]. Available: http://ieeexplore.ieee.org/xpl/articleDetails. jsp?arnumber $=5769557$

[14] G. Wunder, P. Jung, M. Kasparick, T. Wild, F. Schaich, Y. Chen, S. T. Brink, I. Gaspar, N. Michailow, A. Festag, L. Mendes, N. Cassiau, D. Ktenas, M. Dryjanski, S. Pietrzyk, B. Eged, P. Vago, and F. Wiedmann, "5GNOW: non-orthogonal, asynchronous waveforms for future mobile applications," IEEE Communications Magazine, vol. 52, no. 2, pp. 97-105, February 2014

[15] N. Michailow, M. Matth, I. S. Gaspar, A. N. Caldevilla, L. L. Mendes, A. Festag, and G. Fettweis, "Generalized frequency division multiplexing for 5th generation cellular networks," IEEE Transactions on Communications, vol. 62, no. 9, pp. 3045-3061, Sept 2014

[16] A. Farhang, N. Marchetti, and L. E. Doyle, "Low-complexity modem design for GFDM," IEEE Transactions on Signal Processing, vol. 64, no. 6, pp. 1507-1518, March 2016.

[17] M. K. Samimi and T. S. Rappaport, "3-D statistical channel model for millimeter-wave outdoor mobile broadband communications," in 2015 IEEE International Conference on Coтmunications (ICC), June 2015, pp. 2430-2436. 